The University of Southern Mississippi

\title{
The Aquila Digital Community
}

\author{
Faculty Publications
}

8-21-2015

\section{Functional, Sub-100 nm Polymer Nanoparticles via Thiol-ene Miniemulsion Photopolymerization}

\author{
Douglas Amato \\ University of Southern Mississippi, douglas.amato@usm.edu \\ Dahlia Amato \\ University of Southern Mississippi, dahlia.amato@usm.edu \\ Alex S. Flynt \\ University of Southern Mississippi, alex.flynt@usm.edu \\ Derek L. Patton \\ University of Southern Mississippi, Derek.Patton@usm.edu
}

Follow this and additional works at: https://aquila.usm.edu/fac_pubs

Part of the Biochemistry, Biophysics, and Structural Biology Commons

\section{Recommended Citation}

Amato, D., Amato, D., Flynt, A. S., Patton, D. L. (2015). Functional, Sub-100 nm Polymer Nanoparticles via Thiol-ene Miniemulsion Photopolymerization. Polymer Chemistry, 6(31), 5489-5800.

Available at: https://aquila.usm.edu/fac_pubs/15467

This Article is brought to you for free and open access by The Aquila Digital Community. It has been accepted for inclusion in Faculty Publications by an authorized administrator of The Aquila Digital Community. For more information, please contact Joshua.Cromwell@usm.edu. 
Published in final edited form as:

Chem Commun (Camb). 2015 July 11; 51(54): 10910-10913. doi:10.1039/c5cc03319e.

\title{
Functional, composite polythioether nanoparticles via thiol- alkyne photopolymerization in miniemulsion
}

\author{
Dahlia N. Amato ${ }^{a}$, Douglas V. Amato ${ }^{a}$, Jananee Narayanana ${ }^{a}$, Brian R. Donovan ${ }^{a}$, Jessica R. \\ Douglas $^{\mathrm{a}}$, Susan E. Walley ${ }^{\mathrm{a}}$, Alex S. Flynt ${ }^{\mathrm{b}}$, and Derek L. Patton ${ }^{\mathrm{a}}$ \\ Derek L. Patton: derek.patton@usm.edu \\ aSchool of Polymers and High Performance Materials, University of Southern Mississippi, \\ Hattiesburg, MS 39406 USA

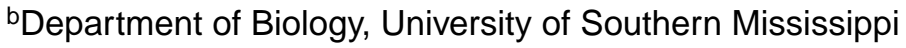

\begin{abstract}
Thiol-yne photopolymerization in miniemulsion is demonstrated as a simple, rapid, and one-pot synthetic approach to polythioether nanoparticles with tuneable particle size and clickable functionality. The strategy is also useful in the synthesis of composite polymer-inorganic nanoparticles.
\end{abstract}

\section{Graphical abstract}

Thiol-yne photopolymerization in miniemulsion is demonstrated as a rapid one-pot synthetic approach to polythioether nanoparticles with tuneable particle size and clickable functionality.

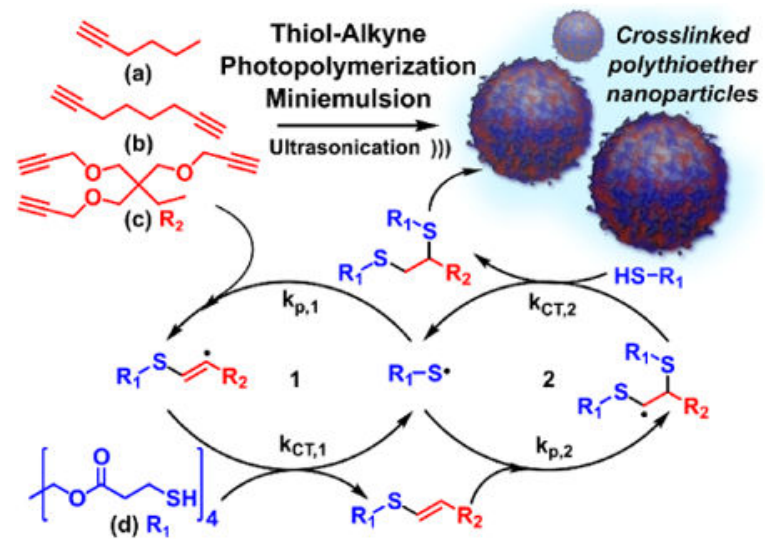

Engineered polymer nanoparticles - with sizes ranging from 20-500 nm - are playing an increasingly important role in the advancement of emerging technologies for industrial, agricultural, pharmaceutical, and biological sectors. Exemplary applications of engineered nanoparticles in these areas include improved agricultural production and crop protection, ${ }^{1}$

Correspondence to: Derek L. Patton, derek . patton@usm. edu.

Electronic Supplementary Information (ESI) available: [Formulation and synthetic details, thermal and spectroscopic characterization]. See DOI: 10.1039/C5CC03319E 
delivery of advanced therapeutics, and bioimaging/biosensing platforms. ${ }^{2}$ Emulsion-based processes - such as miniemulsion polymerizations - provide well-studied synthetic routes to polymer nanomaterials. Miniemulsions polymerizations are characterized as aqueous dispersions of small, narrowly distributed monomer droplets stabilized against Ostwald ripening and collisional degradation by addition of an appropriate surfactant and costabilizer. ${ }^{3}$ Monomer droplets ranging in size from $50-500 \mathrm{~nm}$ are achieved by application of high shear mixing - typically either ultrasonic processing or high-pressure homogenization - and subsequently serve as discrete nanoreactors for the formation of polymer nanoparticles. ${ }^{4}$ Recent miniemulsion literature has focused on "click" polyaddition reactions - such as copper-free or copper-catalysed azide-alkyne 1,3-dipolar cycloaddition $(\mathrm{CuAAC})^{5,6}$ and thiol-mediated chemistries (i.e. thiol-ene ${ }^{7-13}$ and thiol-Michael $\left.{ }^{14}\right)-$ as robust synthetic routes to nanoparticles. Recently, we reported the synthesis of crosslinked polythioether nanoparticles with sub-100 $\mathrm{nm}$ diameters via thiol-ene photopolymerization in miniemulsion. ${ }^{11}$ Additionally, we demonstrated the preparation of nanoparticles with thiol and alkene functional surfaces by exploiting the thiol-ene step polyaddition mechanism under non-stoichiometric monomer feed conditions. The excess thiol and alkene moieties on the nanoparticle surface provided reactive handles for postpolymerization modifications via thiol-Michael and thiol-ene ligation reactions, respectively, to yield fluorescent nanoparticles. However, thiol-ene photopolymerization fails to provide direct access to polymer nanoparticles with one of the most commonly exploited functional groups in the "click" chemistry toolbox - i.e. the alkyne moiety.

Thiol-alkyne photopolymerization provides one such platform to access polymer materials exhibiting alkyne functionality. ${ }^{15-17}$ Thiol-alkyne proceeds via a radical-mediated stepgrowth mechanism involving the addition of two thiols across the alkyne; the first addition yields a vinyl sulfide intermediate that subsequently reacts with a second equivalent of thiol to give the dithioether adduct (Scheme 1). Thiol-alkyne photopolymerization proceeds at room temperature, in the presence of oxygen, with rapid reaction kinetics, and yields inherently thiol or alkyne functional materials resulting from the step-growth process particularly if carried out under non-stoichiometric monomer ratios. ${ }^{17}$ In comparison to thiol-ene, thiol-yne typically provides access to materials with higher crosslink densities and improved thermal properties. ${ }^{18}$ However, thiol-yne photopolymerization has rarely been exploited for functional particle-based platforms. DuPrez et al. ${ }^{19,20}$ first applied this concept for synthesis of thiol or alkyne-functionalized microbeads (diameters $\approx 400 \mu \mathrm{m}$ ) via microfluidics using stoichiometric excess of pentaerythritol tetra(3-mercaptopropionate) (PETMP) or 1,7-octadiyne, and explored the microbeads as resin supports for solid phase synthesis. Aside from DuPrez's microbead work, we are currently unaware of any methodologies reported in literature that exploit thiol-yne photopolymerization for direct synthesis of functional polymer nanoparticles.

Herein, we report thiol-yne photopolymerization in miniemulsion as a simple, rapid, and one-pot synthetic approach to polythioether nanoparticles with tuneable particle size, clickable functionality, and composite compositions. We demonstrate the synthesis of nanoparticles with mean particle diameters ranging from $45 \mathrm{~nm}$ to $200 \mathrm{~nm}$ through simple modifications to the miniemulsion formulation and processing parameters. Facile access to thiol or alkyne functional nanoparticles, and subsequent postpolymerization modifications of 
these functional moieties using thiol-Michael, thiol-yne, and CuAAC click reactions are reported. As shown in Scheme 1, thiol-alkyne miniemulsions were prepared from combinations of pentaerythritol tetra(3-mercaptopropionate) with three different alkyne monomers, including 1-hexyne, 1,7-octadiyne, and trimethylolpropane tripropargyl ether (TMPTPE) to provide polythioether nanoparticles with a range of thermal properties. Hexadecane, Irgacure $184^{\circledR}$ (1-hydroxycyclohexyl phenyl ketone), 4-methoxyphenol, and butyl acetate (BA) served as the hydrophobe, photoinitiator, radical inhibitor (to suppress polymerization during ultrasonication), ${ }^{11}$ and organic diluent, respectively. The organicsoluble constituents were dispersed into the aqueous continuous phase containing sodium dodecylsulfate (SDS) as a surfactant using ultrasonic emulsification. Exposure of these thiolyne miniemulsions to UV light resulted in complete conversion of the thiol and alkyne functional groups, as indicated by the absence of peaks at $2567 \mathrm{~cm}^{-1}$ and $3285 \mathrm{~cm}^{-1}$ in FTIR, respectively (Fig. S1). The size of the dispersed monomer droplets, and consequently the size of the polymer nanoparticles obtained following photopolymerization, depends on a variety of parameters including surfactant concentration, monomer weight fraction, and total ultrasonic energy input. These parameters were explored thoroughly in our recent thiol-ene miniemulsion work; here, we report thiol-yne nanoparticle synthesis under optimized conditions. Figure 1 shows the dependence of nanoparticle size on the monomer phase weight fraction in a miniemulsion formulation containing a fixed amount of surfactant (20 mM SDS). Hexyne, octadiyne, and TMPTPE, when paired with PETMP, all exhibited a minimum particle size of $40-75 \mathrm{~nm}$ between 2 and $3 \mathrm{wt} . \%$ monomer phase - a result that can be attributed to an optimum surface coverage of SDS necessary to stabilize the equilibrium droplet size under these specific conditions. An increase in monomer phase loading depletes SDS coverage enabling droplet coalescence, whereas a decrease in monomer phase loading provides excess SDS that can facilitate Ostwald ripening via the diffusion of organic soluble constituents from smaller droplets, across the aqueous phase, into larger droplets. Both of these conditions resulted in larger nanoparticles, as shown by the u-shaped data in Figure 1a. Nonetheless, low polydispersity values were observed across the monomer loading range, from 0.260 for $2.5 \mathrm{wt} . \%$ to 0.467 for $5 \mathrm{wt} . \%$, as illustrated by the DLS distribution curves in Figure 1b.

The thermal properties of the nanoparticles were analysed by differential scanning calorimetry (DSC). As shown in Fig. S2, hexyne-PETMP nanoparticles exhibited the lowest glass transition temperature $\left(\mathrm{T}_{\mathrm{g}}\right)$ at $-32.5^{\circ} \mathrm{C}$ - a result attributed to a low crosslink density obtained from the monofunctional alkyne. As expected, increasing the functionality of the alkyne to difunctional or trifunctional by employing either 1,7-octadiyne or TMPTPE, respectively, provided nanoparticles with higher $\mathrm{T}_{\mathrm{g}}$. The 1,7-octadiyne based nanoparticles showed a $\mathrm{T}_{\mathrm{g}}$ at $45.7^{\circ} \mathrm{C}$, while TMPTPE based nanoparticles showed a $\mathrm{T}_{\mathrm{g}}$ at $47.3^{\circ} \mathrm{C}$ (Fig. $\mathrm{S} 2$ ). These results are consistent with an expected increase in $\mathrm{T}_{\mathrm{g}}$ with an increase in network crosslink density at higher alkyne functionality.

Particle morphology was characterized using atomic force microscopy (AFM) and transmission electron microscopy (TEM). All samples showed particle sizes in good agreement with data obtained by dynamic light scattering. For the hexyne-PETMP monomer pair (Fig. 2a), the particles exhibited an ill-defined spherical morphology with a strong tendency to aggregate upon drying for analysis. We attribute this behaviour to a low 
crosslink density resulting from the hexyne-PETMP constituents, and consequently a low $T_{g}$ as confirmed by DSC. The low $\mathrm{T}_{\mathrm{g}}$ of these nanoparticles conferred tackiness and led to agglomeration of the particles. However, both the 1,7-octadiyne-PEMTP and TMPTPEPETMP monomer pairs provide nanoparticles with well-defined spherical morphologies that are stable against aggregation upon drying, and could be re-dispersed into aqueous solution. The stability of these nanoparticles can be attributed to the higher glass transition temperature, as discussed previously.

Miniemulsion polymerization offers a versatile approach to synthesize composite inorganicorganic nanoparticles via encapsulation of inorganic materials to endow properties such as magnetism, antimicrobial activity, and fluorescence. ${ }^{12,21-23}$ However, surprisingly few examples have been reported that exploit the rapid nature of photopolymerization to prepare hybrid nanoparticles. ${ }^{24}$ Here, we demonstrate thiol-yne photopolymerization as a rapid twostep synthetic approach to prepare silver/polythioether nanoparticles. First, hydrophobically modified AgNPs were prepared via sodium borohydride reduction of silver nitrate in the presence of dodecanethiol, which yielded $9 \pm 3 \mathrm{~nm}$ AgNPs with a $\lambda_{\max }=435 \mathrm{~nm}$ (Fig. S3 and S4) ${ }^{25}$ After purification, the AgNPs were dispersed in BA and combined with the thiolalkyne monomer formulation. The reaction mixture was then ultrasonicated in the presence of water and SDS, and polymerized with ultraviolet light for 20 min to yield composite $\mathrm{Ag}$ / polythioether nanoparticles. This photopolymerization methodology markedly improves upon current thermal miniemulsion routes, which typically require $4-24 \mathrm{~h}$ reaction time to yield composite nanoparticles. ${ }^{22,23}$ TEM analysis revealed well-defined core-shell particle morphologies with AgNPs strictly confined within the core of the polythioether nanoparticles (Fig. 3a). Image analysis carried out on a population of nanoparticles imaged at $50 \mathrm{keV}$ revealed an average composite diameter of $127 \pm 8 \mathrm{~nm}$, an average inorganic core diameter of $68 \pm 6 \mathrm{~nm}$, and a clearly defined polythioether shell of $\sim 30 \mathrm{~nm}$. Additional TEM images collected at $200 \mathrm{keV}$ showed that the inorganic core was comprised of multiple individual AgNPs (Fig. 3b). It is noteworthy that relatively few "empty" polythioether nanoparticles (i.e. devoid of AgNPs in the core) or unencapsulated AgNPs were observed in the TEM images surveyed - an observation indicative of a high encapsulation efficiency that minimizes the need for subsequent purification protocols.

To take full advantage of the step polyaddition nature of thiol-yne photopolymerization, different stoichiometries of thiol (SH) and alkyne were reacted within the miniemulsions to prepare thiol or alkyne functionalized polymer nanoparticles. The ratio of SH to alkyne were adjusted from 1.5:1 and 3.2:1, and the resulting nanoparticles were analysed via FTIR (Fig. S1). Nanoparticles prepared from the monomer feed with excess SH (3.2:1 SH:yne) showed the presence of residual thiol functionality at $2567 \mathrm{~cm}^{-1}$. Conversely, nanoparticles resulting from the monomer feed with excess yne (1.5:1, SH:yne) showed a strong alkyne absorption at $3285 \mathrm{~cm}^{-1}$. The preservation of the excess thiol and alkyne functionality provided a convenient strategy for postpolymerization modification of the nanoparticle surface using various click reactions. As illustrated in Scheme 2, thiol-yne, thiol-Michael, and CuAAC reactions were employed to ligatea series of fluorescent dyes to the nanoparticle surface. To the thiol-functionalized nanoparticle (3.2:1 SH:yne), Texas Red maleimide was attached using a thiol Michael click reaction (Scheme 2a). Following purification by repetitive 
centrifugation/wash steps, nanoparticles with red fluorescence were confirmed by confocal microscopy $\left(\lambda_{\text {em }}\right.$ at $615 \mathrm{~nm}$, Fig. 4a).

The alkyne-functionalized nanoparticles were tagged with fluorescent dyes via two routes. First, 7-mercapto-4-methylcoumarin was immobilized using a photoinitiated thiol-yne reaction in the presence of 2,2-dimethoxy-2-phenylacetophenone to afford nanoparticles that fluoresce blue ( $\lambda_{\mathrm{em}}$ at $385 \mathrm{~nm}$ ), as shown by confocal microscopy in Fig. 4b. Lastly, the CuAAC click reaction between Alexa Fluor ${ }^{\circledR} 488$ Azide and the alkyne-functionalized nanoparticles resulted in fluorescently tagged nanoparticles with green emission $\left(\lambda_{\mathrm{em}}\right.$ at 385 nm, Fig. 4c). Control experiments were also carried out under the same conditions using non-reactive dyes to show physisorption plays no role in immobilization of the fluorescent tags (Fig. 4d and S5). This two-step process of generating functional nanoparticles and subsequent functionalization through high efficiency reactions simplifies current multisynthetic processes while also expanding the library of functional groups that can react with these particles.

In conclusion, we have demonstrated the versatility of thiol-alkyne photopolymerization in miniemulsion for the preparation of polythioether nanoparticles. Simple off-stoichiometric monomer feed ratios provided access to functional nanoparticles that express thiol and alkyne moieties at the nanoparticle surface - and these moieties are readily available for postpolymerization modification using various click chemistries. We also demonstrated thiol-yne photopolymerization in miniemulsion as a means to synthesize hybrid silver/ polythioether nanoparticles with well-defined core-shell morphologies; this approach provides hybrid nanoparticles in a fraction of time $(20 \mathrm{~min})$ as compared with previously reported thermally-initiated routes (4-24 h). We anticipate that thiol-yne miniemulsions will provide facile access to a functional and hybrid nanoparticle platform with antimicrobial, delivery, and imaging applications.

\section{Supplementary Material}

Refer to Web version on PubMed Central for supplementary material.

\section{Acknowledgments}

We wish to acknowledge financial support from the National Science Foundation (DMR-1056817, IIA-1430364, and DGE-1449999). D.N.A. acknowledges fellowship support from the NSF GK-12 program "Molecules to Muscles" (Award \#0947944) through the University of Southern Mississippi. B.R.D. thanks the US Dept. of Education GAANN Fellowship Program (Award \# P200A120118) for financial support. We thank Mark Brei from the Storey Group for providing TMPTPE. Confocal microscopy was supported by MS INBRE funded by NCRR (5P20RR-016476-11) and NIGMS/NIH (8 P20 GM103476-11).

\section{Notes and references}

1. Khot LR, Sankaran S, Maja JM, Ehsani R, Schuster EW. Crop Protection. 2012; 35:64-70.

2. Petros RA, DeSimone JM. Nat Rev Drug Discov. 2010; 9:615-627. [PubMed: 20616808]

3. Asua JM. Prog Polym Sci. 2002; 27:1283-1346.

4. Landfester K. Angew Chem Int Ed. 2009; 48:4488-4507.

5. Roux R, Sallet L, Alcouffe P, Chambert S, Sintes-Zydowicz N, Fleury E, Bernard J. ACS Macro Lett. 2012; 1:1074-1078. 
6. Siebert JM, Baier G, Musyanovych A, Landfester K. Chem Commun. 2012; 48:5470-5472.

7. Durham OZ, Krishnan S, Shipp DA. ACS Macro Lett. 2012; 1:1134-1137.

8. Durham OZ, Shipp DA. Polymer. 2014; 55:1674-1680.

9. Jasinski F, Lobry E, Tarablsi B, Chemtob A, Croutxé-Barghorn C, Le Nouen D, Criqui A. ACS Macro Lett. 2014; 3:958-962.

10. Tan J, Li C, Zhou J, Yin C, Zhang B, Gu J, Zhang Q. RSC Adv. 2014; 4:13334.

11. Amato DV, Amato DN, Flynt AS, Patton DL. Polym Chem. 201510.1039/C1034PY01449A

12. van Berkel KY, Hawker CJ. J Polym Sci Part A: Polym Chem. 2010; 48:1594-1606.

13. Zou J, Hew CC, Themistou E, Li Y, Chen CK, Alexandridis P, Cheng C. Adv Mater. 2011; 23:4274-4277. [PubMed: 22039596]

14. Wang C, Chatani S, Podgorski M, Bowman CN. Polym Chem. 2015; 6:3758-3763.

15. Chan JW, Zhou H, Hoyle CE, Lowe AB. Chem Mater. 2009; 21:1579-1585.

16. Lowe AB, Hoyle CE, Bowman CN. J Mater Chem. 2010; 20:4745-4750.

17. Fairbanks BD, Scott TF, Kloxin CJ, Anseth KS, Bowman CN. Macromolecules. 2009; 42:211217. [PubMed: 19461871]

18. Chan JW, Shin J, Hoyle CE, Bowman CN, Lowe AB. Macromolecules. 2010; 43:4937-4942.

19. Gokmen MT, Brassinne J, Prasath RA, Du Prez FE. Chem Commun. 2011; 47:4652-4654.

20. Prasath RA, Gokmen MT, Espeel P, Du Prez FE. Polym Chem. 2010; 1:685-692.

21. Erdem B, Sudol ED, Dimonie VL, El-Aasser MS. J Polym Sci Part A: Polym Chem. 2000; 38:4419-4430.

22. Ramírez LP, Landfester K. Macromol Chem Phys. 2003; 204:22-31.

23. van Berkel KY, Piekarski AM, Kierstead PH, Pressly ED, Ray PC, Hawker CJ. Macromolecules. 2009; 42:1425-1427.

24. Liu D, Jiang X, Yin J. Langmuir. 2014; 30:7213-7220. [PubMed: 24917521]

25. Farrell Z, Shelton C, Dunn C, Green D. Langmuir. 2013; 29:9291-9300. [PubMed: 23855440] 


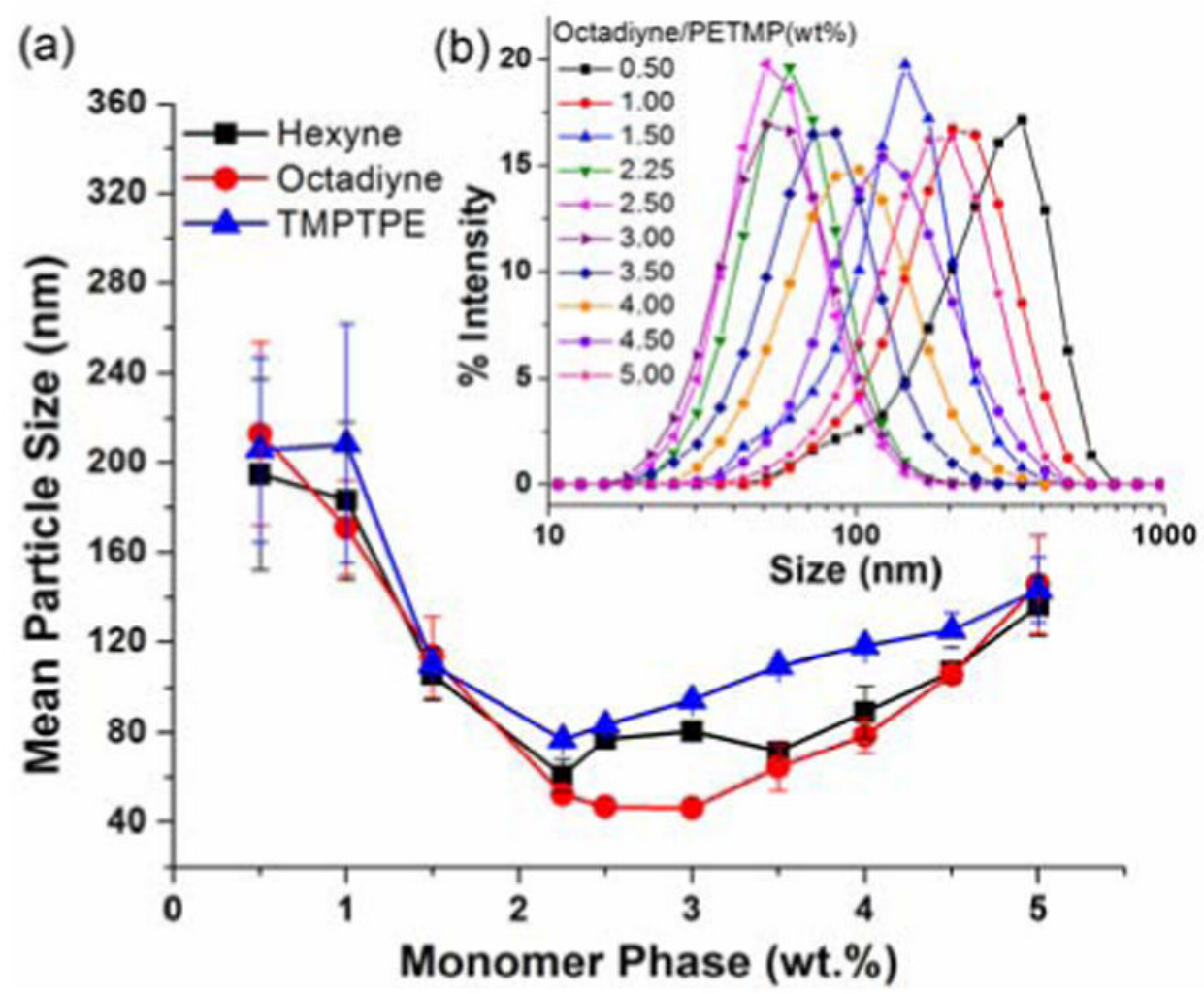

Figure 1.

(a) Effect of weight fraction of the organic monomer phase on particle size. (b) Inset shows nanoparticle size distribution curves obtained by dynamic light scattering. (Synthetic conditions: $20 \mathrm{~min}, 20 \%$ amplitude ultrasonication, $10 \mathrm{~min}$ UV exposure). 

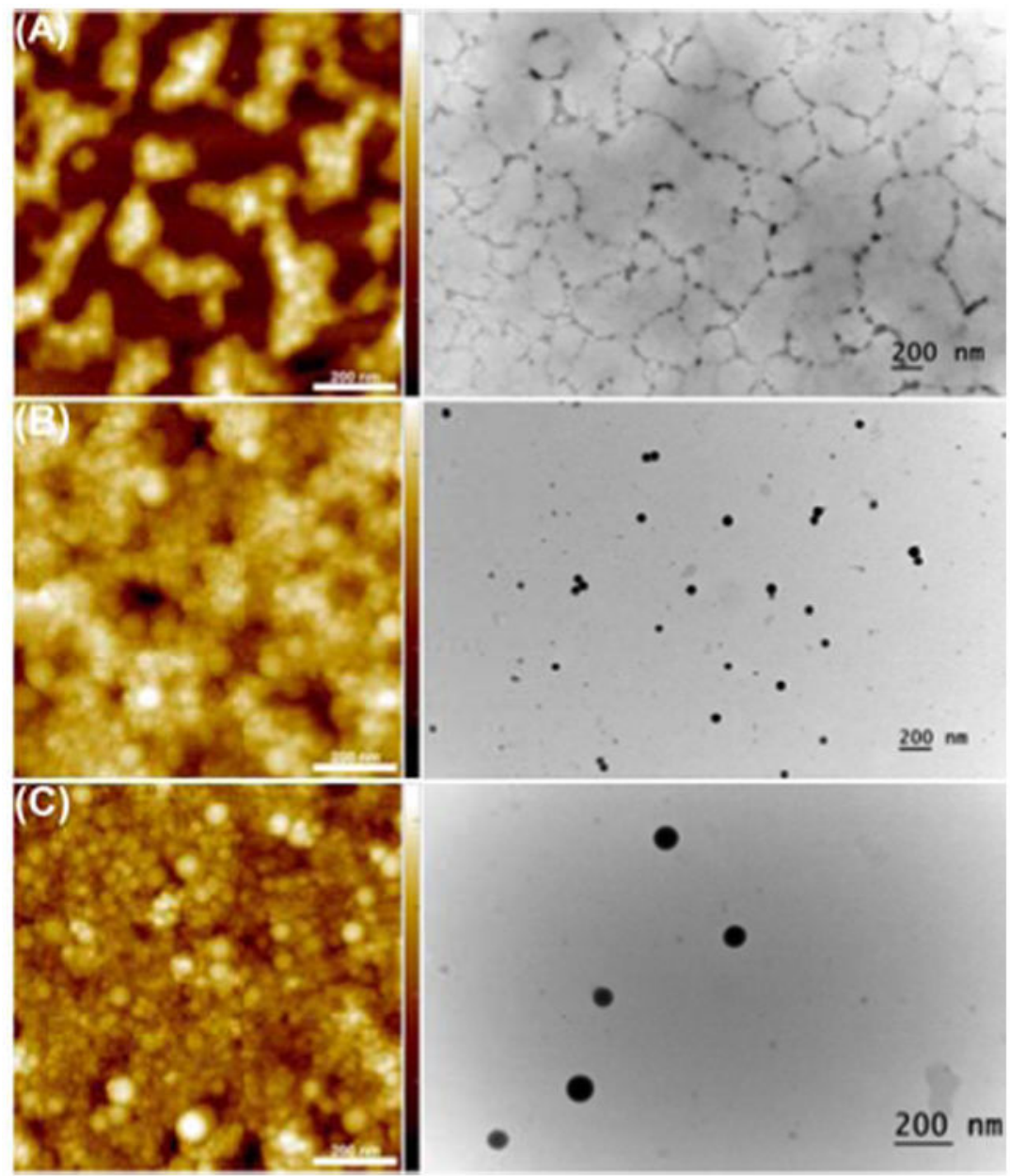

$\bullet$

$\underline{200} \mathrm{~nm}$

Figure 2.

AFM and TEM images corresponding to (A) hexyne-PETMP, (B) 1,7-octadiyne-PETMP, and (C) TMPTPE-PETMP particles. All scale bars are $200 \mathrm{~nm}$. 


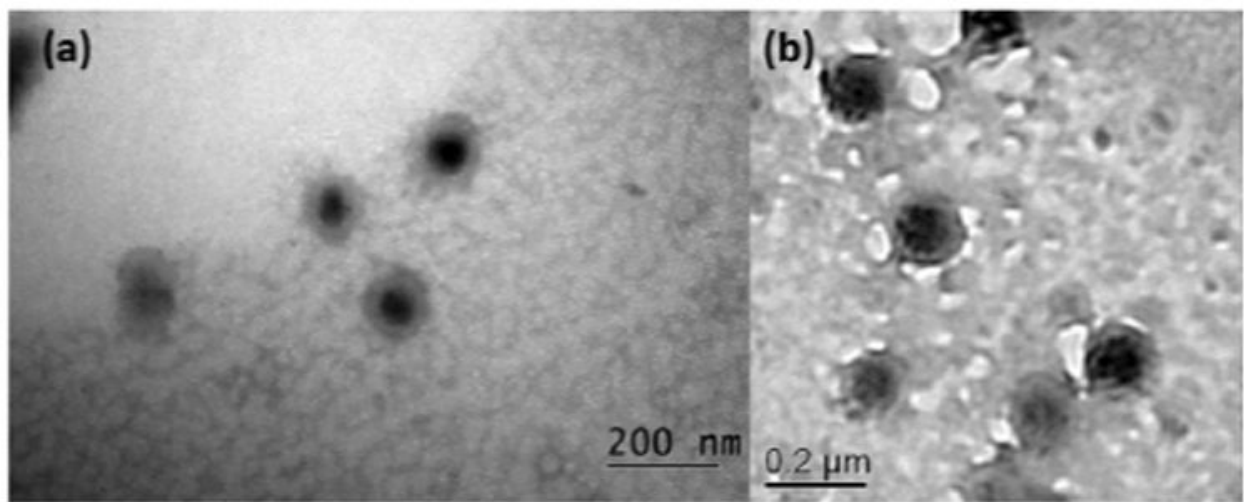

Figure 3.

Representative TEM micrographs of composite polythioether-silver nanoparticles collected at (a) $50 \mathrm{keV}$ and (b) $200 \mathrm{keV}$, showing clusters of $9 \mathrm{~nm} \mathrm{AgNPs}$ encapsulated within 1,7octadiyne-PETMP nanoparticles. 


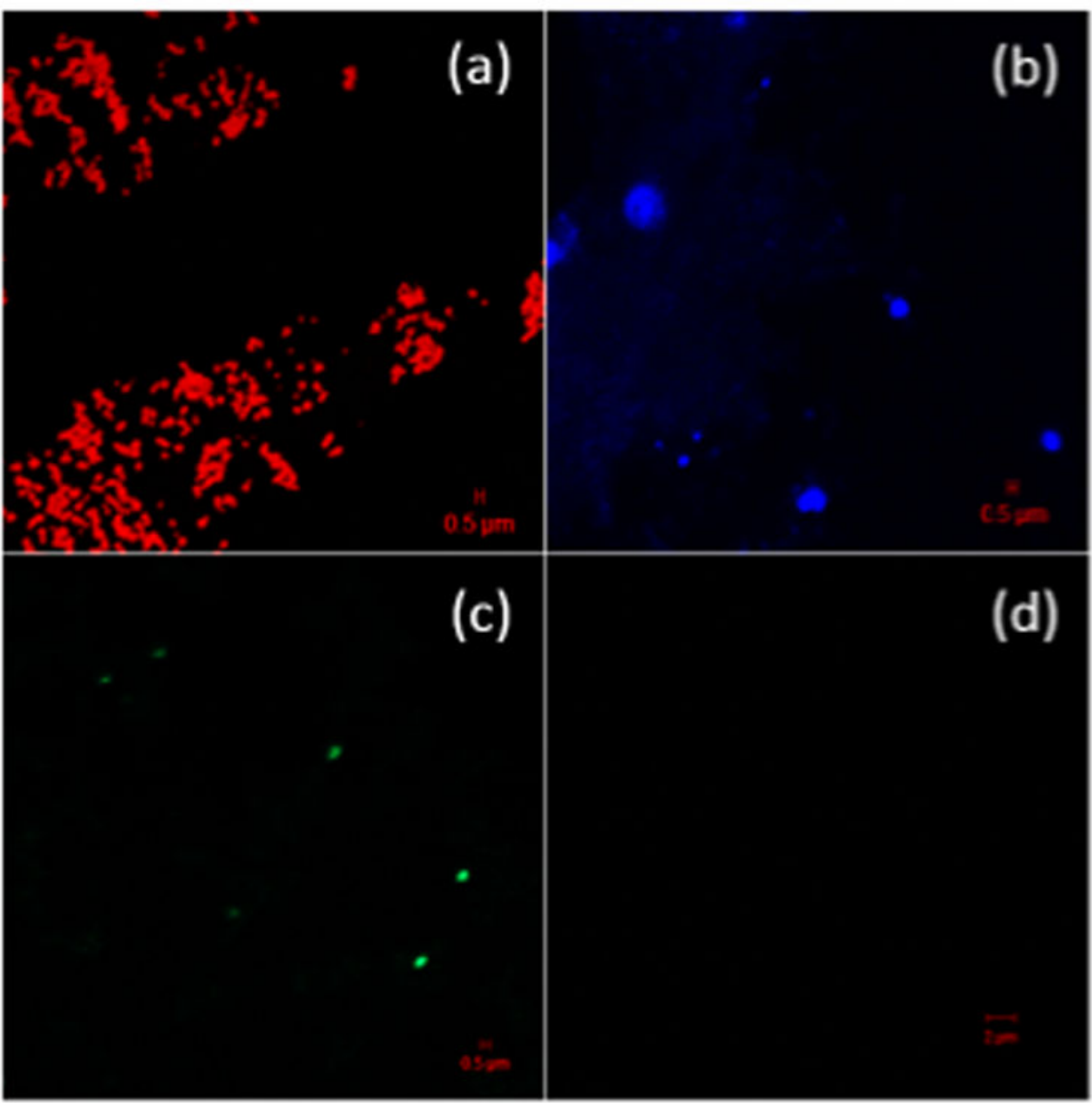

Figure 4.

Fluorescence microscopy of (a) thiol-functional nanoparticles postmodified with Texas Red maleimide using a thiol-Michael reaction, (b) alkyne-functional nanoparticles postmodified by photoinitiated thiol-yne with 7-mercapto-4-methylcoumarin, and (c) alkyne-functional nanoparticles postmodified by CuAAC with Alexa Fluor ${ }^{\circledR} 488$ Azide. (d) shows a control experiment with non-reactive dyes. 
<smiles>C#CCCCC</smiles>

(a)

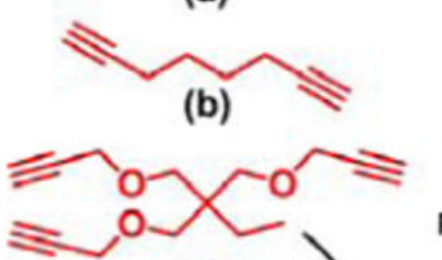

(a), (b), or (c) with (d), surfactant, $\mathrm{HD}$, $\mathrm{BA}_{\text {disp, }} \mathrm{H}_{2} \mathrm{O}_{\text {cont }}$

Ultrasonication I)!

$$
\mathrm{UV}_{3 \mathrm{3esm}}
$$

Photopolymerization

(c) $R_{2}$
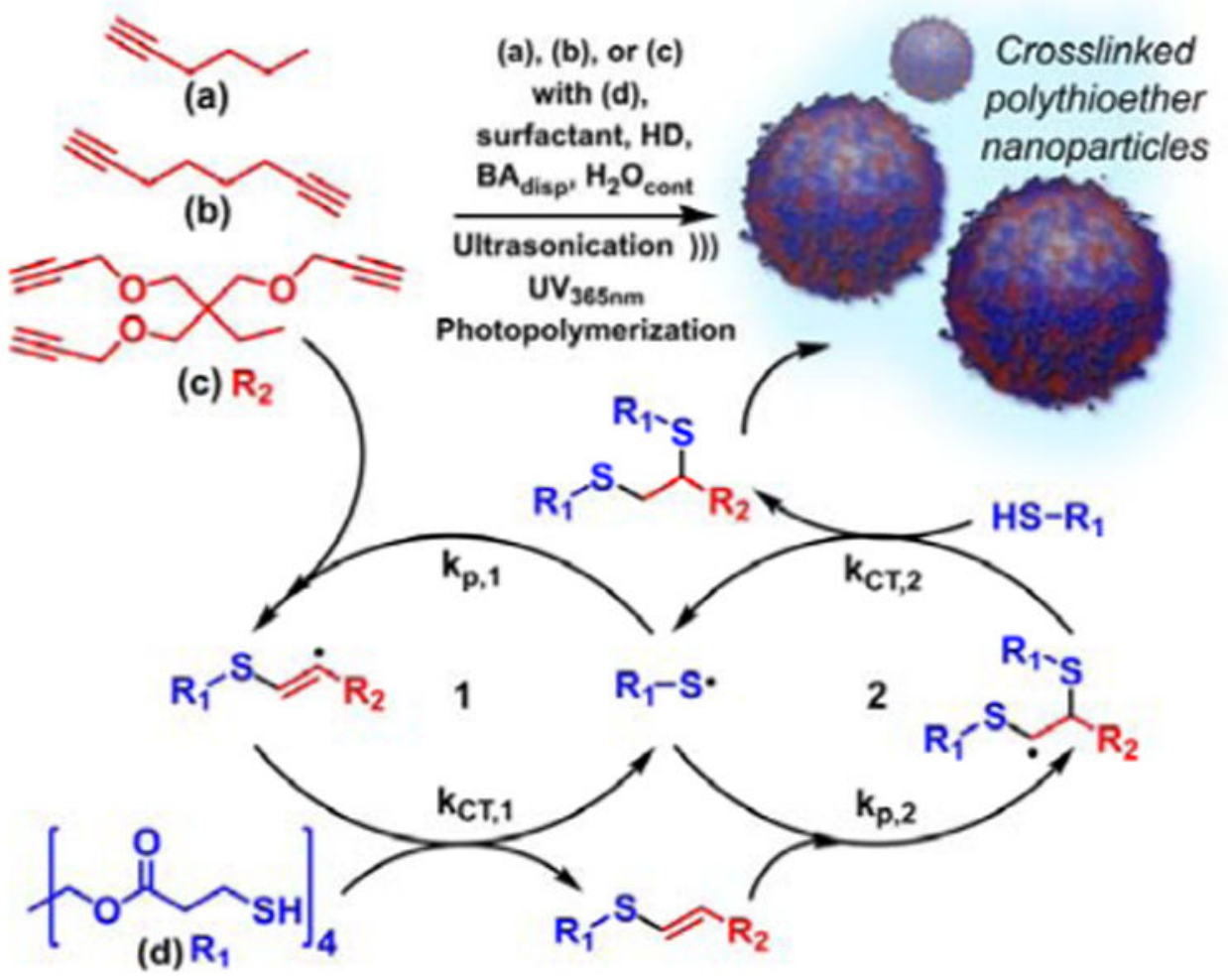

Scheme 1.

Various multifunctional alkynes (a-c) and tetrafunctional thiol (d) used to generate polythioether nanoparticles via thiol-alkyne photopolymerization in miniemulsion. Thiolyne involves sequential addition and hydrogen abstraction steps of primary alkynes (1) and subsequent vinyl sulfides (2) to generate crosslinked nanoparticles. 

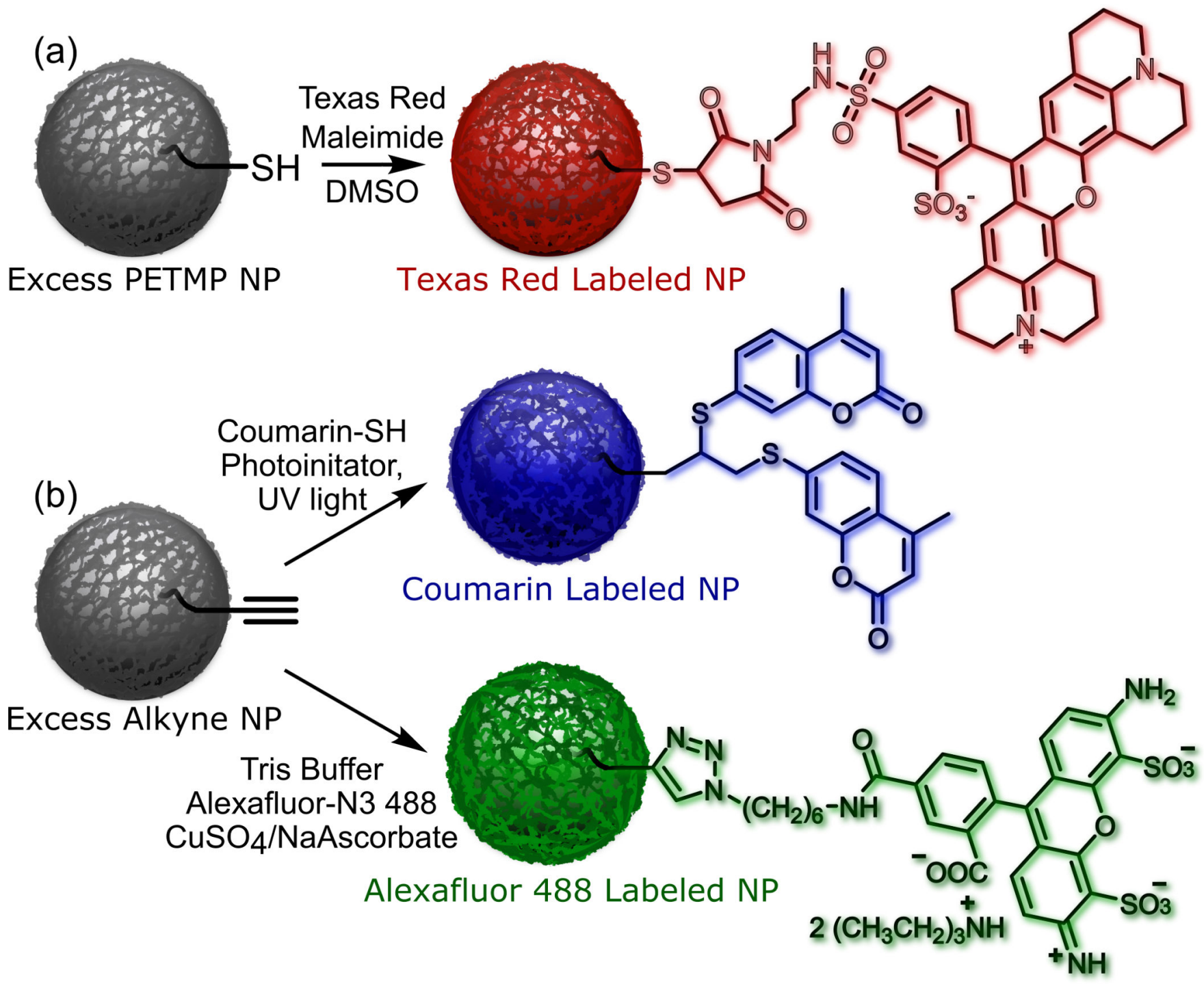

Scheme 2.

(a) Thiol-functional polythioether nanoparticles prepared with excess PETMP and postmodified via thiol-Michael with Texas Red maleimide. (b) Alkyne-functional polythioether nanoparticles prepared with excess 1,7-octadiyne postmodified with 7mercapto-4-methylcoumarin via thiol-yne or with Alexa Fluor ${ }^{\circledR} 488$ Azide via CuAAC. 